\title{
Variable aperture horn antenna for millimeter wave wireless communications
}

\author{
Edward Lewis, Laxma R. Billa, Rosa Letizia Senior Member, IEEE, Claudio Paoloni, Senior Member, IEEE \\ Engineering Department, Lancaster University \\ Lancaster, UK, LA1 4YW
}

\begin{abstract}
A variable aperture horn antenna, for flexible coverage area in point to multipoint at millimeter wave, is proposed. The antenna is intended to be implement in future $5 \mathrm{G}$ high capacity wireless backhaul networks to respond to traffic variations and coverage needs. Design and test results of the variable aperture antenna at scaled 35 - $40 \mathrm{GHz}$ frequency band are reported.
\end{abstract}

Keywords - Horn antenna, Variable aperture, 5G, Wireless communication, Backhaul

\section{INTRODUCTION}

Antennas are important components for assuring the correct coverage in wireless communication. Point to multipoint wireless distribution is based on a transmission hub with a low gain antenna to cover a given area sector where a number of terminals are arbitrarily deployed [1]. European Commission H2020 TWEETHER "Travelling wave tube based w-band wireless networks with high data rate distribution, spectrum \& energy efficiency" project develop the first point to multipoint wireless system at Wband for backhaul and fixed access [2-3]. However, the future $5 \mathrm{G}$ wireless backhaul systems demand flexible coverage to respond to the traffic variations. Such a requirements can be fulfilled by automatically adjustment of the horn aperture to produce a range of aperture angles, maintaining high quality radiation properties [4-6].

In this paper a variable gain and aperture angle horn antenna based on a movable ridge in antenna body is proposed. This proposed antenna is designed for use in the W-band TWEETHER system. As proof of concept, a first design and test have been made at Ka-band.

\section{Design AND Simulation}

The variation of the horn antenna gain in the H-plane is function of the dimension Ax (Fig.1). To vary this dimension, without changing the body of the antenna, a metal sheet supported by two tuning screws, that can be moved by a microcontroller, is added to modify the antenna geometry to change the H-plane gain, maintaining the E-plane gain unaffected. The variable aperture horn antenna is shown in Figure 1.

CST-Microwave studio is used to design and simulated the proposed antenna in the frequency range $36-40 \mathrm{GHz}$. Figure 2 shows the radiation patterns for three different positions of

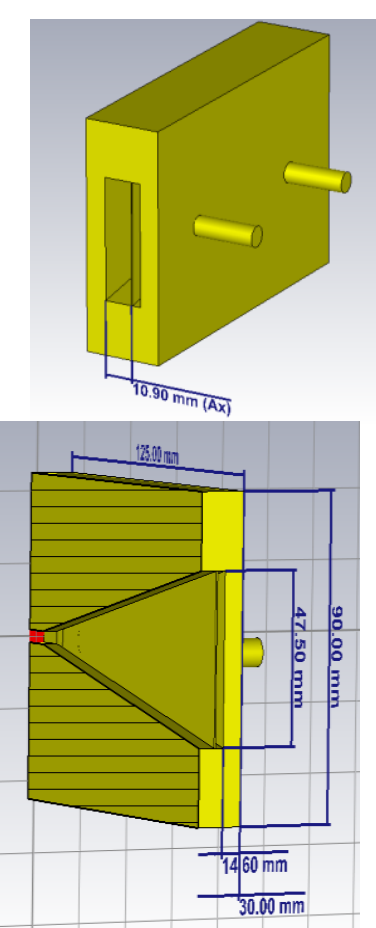

Fig. 1. Variable aperture horn antenna.

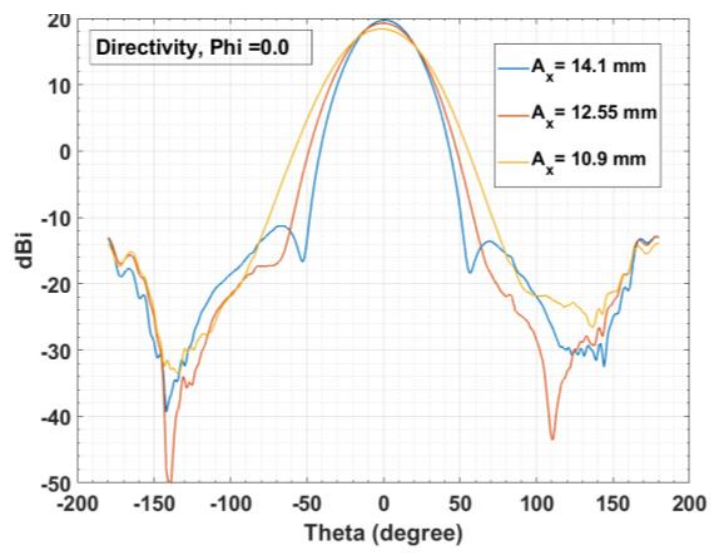

Fig. 2. 2D radiation pattern of the variable horn with adjustable aperture depths $\left(A_{x}\right)$ of $14.1 \mathrm{~mm}, 12.55 \mathrm{~mm}$ and $10.9 \mathrm{~mm}$. 
the ridge. The three aperture angles, $35^{\circ}, 40^{\circ}$ and $45^{\circ}$ corresponding to $18.5 \mathrm{dBi}, 19.3 \mathrm{dBi}$ and $19.8 \mathrm{dBi}$ antenna gain respectively, are achieved. The position of the adjustable metal sheet is $14.1 \mathrm{~mm}, 12.55 \mathrm{~mm}$, and $10.9 \mathrm{~mm}$ respectively. The radiation patterns demonstrated excellent directivity with low side lobes for minimizing the interference.

\section{MAnufactruing AND Testing}

To validate the proposed design, a prototype of variable aperture horn antenna was manufactured by using CNC milling in Aluminum (Fig.3).

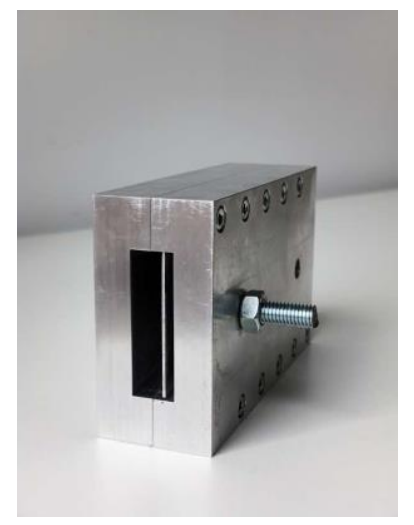

Fig. 3. Variable aperture horn antenna

A Rohde \& Schwarz Vector Network Analyzer (VNA) was used to measure the reflection coefficient, $S_{11}$, of the manufactured antenna. The results clearly demonstrated very good performance in the $33 \mathrm{GHz}-40 \mathrm{GHz}$ frequency range, with $S_{11}$ better than $-15 \mathrm{~dB}$ (fig. 4). This first prototype antenna demonstrates the validity of the approach based on the

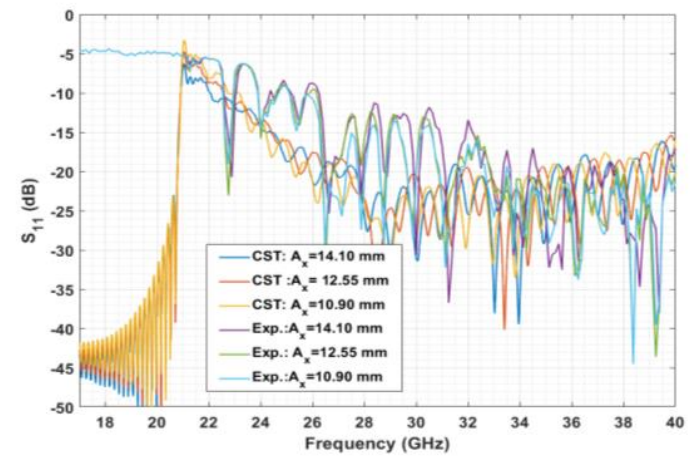

Fig. 4. Test results of $S_{11}$ of the aperture horn antenna with adjustable aperture depths $\left(\mathrm{A}_{\mathrm{x}}\right)$ of $14.1 \mathrm{~mm}, 12.55 \mathrm{~mm}$ and $10.9 \mathrm{~mm}$. variable position metal sheet for achieving different apertures. The antenna gain was measured in far-field from a reference antenna. The far-field was taken to start at a distance $2 \mathrm{D}^{2} / \lambda_{0}$, where $\mathrm{D}$ is its larger dimensions at the opening of the reference horn, and $\lambda_{0}$ is the free-space wavelength. Wave reflections from the ground and other objects were not considered, as it is amount of power reflected to the test antenna minimal. Figure 5 shows the measured gain of the test antenna at a far-field distance of $40 \mathrm{~cm}$ from the reference antenna. It was noted that the gain is about $20 \mathrm{~dB}$ between the frequency $33-37.5 \mathrm{GHz}$.

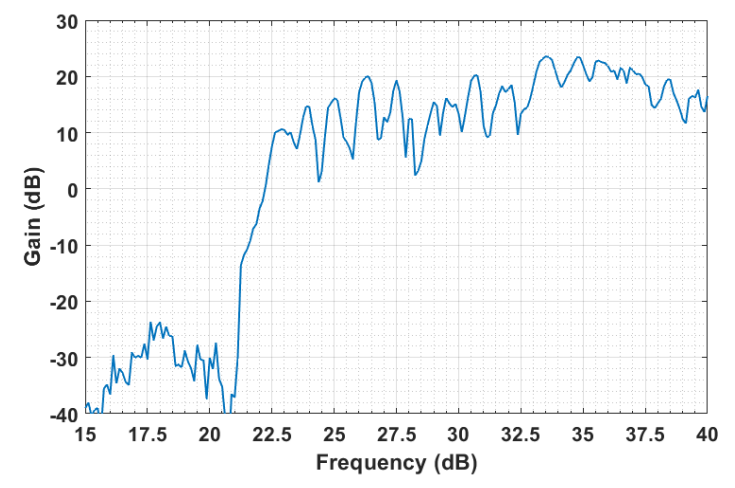

Fig. 5. Gain of the test antenna when $A_{x}=14.1 \mathrm{~mm}$.

\section{CONCLUSION}

A novel variable aperture horn antenna has been successfully designed and tested. This antenna would provide a flexible coverage by variable angle sector remotely controlled.

\section{ACKNOWLEDGMENT}

The authors acknowledged Jonathan Gates for the great mechanical fabrication support.

\section{REFERENCES}

[1] Omar, Muhammad Shahmeer, et al. "Performance analysis of hybrid 5G cellular networks exploiting mmWave capabilities in suburban areas." 2016 IEEE International Conference on Communications (ICC). IEEE, 2016.

[2] www.tweether.eu

[3] Paoloni, Claudio, et al. "A Traveling Wave Tube for 92-95 GHz band wireless applications." $201641^{\text {st }}$ International Conference on Infrared, Millimeter, and Terahertz waves (IRMMW-THz). IEEE, 2016.

[4] Abbas-Azimi, M., et al. "Design and optimization of a new 1-18 GHz double ridged guide horn antenna." Journal of Electromagnetic Waves and Applications 21.4 (2007): 501-516.

[5] Mallahzadeh, Ali Reza Reza, Ali Akbar Dastranj, and Hamid Reza Hassani. "A novel dual-polarized double-ridged horn antenna for wideband applications." Progress In Electromagnetics Research 1 (2008): 67-80.

[6] Jouade, Antoine, et al. "Mechanically pattern-reconfigurable bended horn antenna for high-power applications." IEEE Antennas and Wireless Propagation Letters 16 (2016): 457-460. 